\title{
Comparison of medication adherence and persistence in type 2 diabetes: A systematic review and meta-analysis
}

\author{
Andrew McGovern BMBS $\odot$ | Zayd Tippu MBBS | William Hinton MSc | Neil Munro PhD | \\ Martin Whyte PhD | Simon de Lusignan MD
}

Section of Clinical Medicine and Aging, Department of Clinical and Experimental Medicine, University of Surrey, Guildford, UK

\section{Correspondence}

Andrew McGovern, Section of Clinical

Medicine and Aging, Department of Clinical and Experimental Medicine, Austin Pearce Building, University of Surrey, Guildford, GU2 7PX, UK.

Email: a.mcgovern@surrey.ac.uk

Funding information

Funding for this study was provided by Eli Lilly and Company. The funders were not involved in the study design, conduct, or manuscript creation, and had no influence over the decision to publish.

\begin{abstract}
Limited medication adherence and persistence with treatment are barriers to successful management of type 2 diabetes (T2D). We searched MEDLINE, EMBASE, the Cochrane Library, the Register of Controlled Trials, PsychINFO and CINAHL for observational and interventional studies that compared the adherence or persistence associated with 2 or more glucose-lowering medications in people with T2D. Where 5 or more studies provided the same comparison, a random-effects meta-analysis was performed, reporting mean difference (MD) or odds ratio (OR) for adherence or persistence, depending on the pooled study outcomes. We included a total of 48 studies. Compared with metformin, adherence (\%) was better for sulphonylureas (5 studies; MD 10.6\%, 95\% confidence interval [Cl] 6.5-14.7) and thiazolidinediones (TZDs; 6 studies; MD $11.3 \%, 95 \% \mathrm{Cl} 2.7 \%-20.0 \%$ ). Adherence to TZDs was marginally better than adherence to sulphonylureas (5 studies; MD 1.5\%, 95\% Cl 0.1-2.9). Dipeptidyl peptidase-4 inhibitors had better adherence than sulphonylureas and TZDs. Glucagon-like peptide-1 receptor agonists had higher rates of discontinuation than long-acting analogue insulins (6 studies; OR 1.95; 95\% Cl 1.17-3.27). Longacting insulin analogues had better persistence than human insulins ( 5 studies; MD 43.1 days; $95 \% \mathrm{Cl} 22.0-64.2)$. The methods used to define adherence and persistence were highly variable.
\end{abstract}

\section{KEYWORDS}

antidiabetic drug, GLP-1 analogue, insulin analogues, medication adherence, medication persistence, meta-analysis, systematic review, type 2 diabetes

\section{1 | INTRODUCTION}

Non-adherence and non-persistence with medications in type 2 diabetes (T2D) are associated with worse outcomes, including poorer glycaemic control, more complications and higher overall costs. ${ }^{1-4}$ Despite these adverse outcomes, a substantial proportion of people with T2D do not take their medication as prescribed. ${ }^{5,6}$

Medication-related factors are a major contributor to nonadherence, ${ }^{7}$ and adherence rates differ considerably across drug classes in chronic diseases. ${ }^{8}$ Identification of any substantial differences in adherence rates between medication classes used in T2D is important to help guide prescribing, maximize treatment adherence, and consequently minimize complications.

Differing terminology has been used to describe patient use of medications, including adherence, compliance, concordance and persistence. ${ }^{9}$ Two discrete patterns of use are described using these terms: "missed medication doses" (adherence, compliance or concordance) and "duration of use before termination or substantial medication gap" (persistence). Whilst "compliance" is the most commonly used term for the former pattern of use, ${ }^{9}$ the term "concordance" is sometimes preferred to emphasize the joint agreement between physician and patient to use the medication in a certain way. ${ }^{10}$ "Adherence" is the term recommended by the World Health Organisation (WHO ${ }^{7}$ and is the term used in the present study. "Persistence with medication" is the preferred terminology for the duration of medication use. ${ }^{9}$

\section{2 | METHODS}

In the present systematic review and meta-analysis we compared adherence and persistence rates across different medication classes for the treatment of hyperglycaemia in people with T2D. We 
attempted to answer the question: Are there substantially different adherence and persistence rates among the different classes of therapies available for T2D? The review protocol has been previously published $^{2}$ and registered with the PROSPERO register of systematic reviews (CRD42015027865). The method is summarized below. All deviations from the original protocol are reported in Appendix S1 in File 1, with the rationale.

\section{1 | Study selection and identification}

Interventional and observational studies providing a measure of adherence to or persistence with medications from 2 or more distinct classes of agent (Table S1 in File 1) in the treatment of T2D were included for analysis.

We searched MEDLINE, Embase, the Cochrane Library, the International Standard Randomized Controlled Trials Number (ISRCTN) registry (www.isrctn.com), PsychINFO and the Cumulative Index to Nursing and Allied Health Literature (CINAHL) for the period January 1, 2006 to November 5, 2015, using a predefined search strategy (Appendix S2 in File 1). Bibliographic searches of the included studies were also performed.

Two reviewers independently performed the searches and reviewed the titles and abstracts of all the articles identified. Where either reviewer considered the study to be of possible relevance, the full text was sought. The reviewers then independently reviewed all full texts and decided on their inclusion or exclusion. A third reviewer made a final decision where there were remaining discrepancies.

\subsection{Data analysis}

Data were extracted independently by 2 reviewers. Study results were combined in our meta-analysis, using the approach described in Appendix S3 in File 1, where $\geq 5$ studies provided a comparison between the same 2 classes and used a comparable outcome measure.

Study quality was assessed against the Newcastle-Ottowa scale (Appendix S4 in File 1) for observational studies. The quality of clinical trials was assessed using the Cochrane risk assessment tool. Two reviewers independently assessed study quality and, where scores were not in agreement, a third opinion was sought from an adjudicator.

\section{3 | RESULTS}

Through electronic database searching we identified 4466 records for title and abstract review and 13 additional studies from bibliographic searching of included articles (Figure S1 in File 1). Forty-eight studies met our inclusion criteria for the final narrative synthesis, with a combined population of 1696939 people with T2D. Of these, 25 studies compared oral therapies only, 19 compared injectable therapies only, and 3 included a comparison between oral and injectable therapies. We also found 1 study which compared an oral with an inhaled agent. Most studies were conducted in North America (USA, 21; Canada, 1; and USA and Mexico, 1), with others in Europe (Germany, 5; UK, 4; Italy, 3; Ireland, 1; Sweden, 1; Netherlands, 1; Hungary, 1; France, 1; multiple European countries, 3), and elsewhere (Iran, 1; multiple countries, 4).

\section{1 | Oral medications}

Twenty-six studies compared oral therapies (Tables S2 and S3 in File 1): 23 observational studies (18 retrospective and 5 prospective cohort studies) and 3 randomized controlled trials (RCTs).

It was possible to compare adherence in a meta-analysis for metformin, sulphonylureas and thiazolidinediones (TZDs; Figure 1 and Figures S2-S6 in File 1). When adherence was compared using mean adherence (\% measured using medication possession ratio [MPR] or proportion of days covered [PDC]) sulphonylureas and TZDs were associated with substantially better adherence than metformin, and TZDs had marginally better adherence than sulphonylureas. When adherence was compared using the odds ratio (OR) for non-adherence, differences were not significant.

Multiple studies compared dipeptidyl peptidase-4 (DPP-4) inhibitors with sulphonylureas and TZDs (although meta-analysis was not possible). DPP-4 inhibitors were found to have better adherence and longer persistence than sulphonylureas and TZDs in almost all studies. Only 1 study examined $\alpha$-glucosidase inhibitors and meglitinides, and reported lower adherence for both classes than for other oral options. One study included a sodium-glucose co-transporter-2 (SGLT2) inhibitor (canagliflozin), which had better persistence than DPP-4 inhibitors.

\section{2 | Injectable medications}

Three studies compared GLP-1 receptor agonists with oral therapies (Table S3 in File 1). These demonstrated shorter persistence with GLP-1 receptor agonists than with DPP-4 inhibitors and lower adherence than sulphonylureas and DPP-4 inhibitors.

Eighteen studies compared multiple injectable therapies (Table S4 in File 1): 12 observational studies (all retrospective cohorts) and 6 RCTs.

In meta-analyses, it was possible to compare the persistence associated with long-acting insulin analogues with that associated with medium-acting human insulins and GLP-1 receptor agonists (Figure 1 and Figures S7 and S8 in File 1). Long-acting insulin analogues had significantly longer persistence in both comparisons. The higher rates of discontinuation with GLP-1 receptor analogues were attributed to adverse effects (predominantly injection site reactions and gastrointestinal disturbance) in several studies.

Only 1 study analysed prandial insulins, comparing persistence between short-acting human insulins and rapid-acting analogue insulins when added to basal insulin. The study found better persistence with analogue insulins. Two observational studies compared both adherence and persistence with mixed insulins with those associated with long-acting analogue insulins. Both studies found longer persistence with analogue insulins, but only 1 found a difference in adherence (lower for premixed insulin).

\section{3 | Inhaled medications}

One study described a pair of RCTs in which inhaled insulin was compared with metformin and the sulphonylurea glibenclamide (Table S5 in File 1). No difference was found between inhaled insulin and either oral therapy in discontinuation rates. 


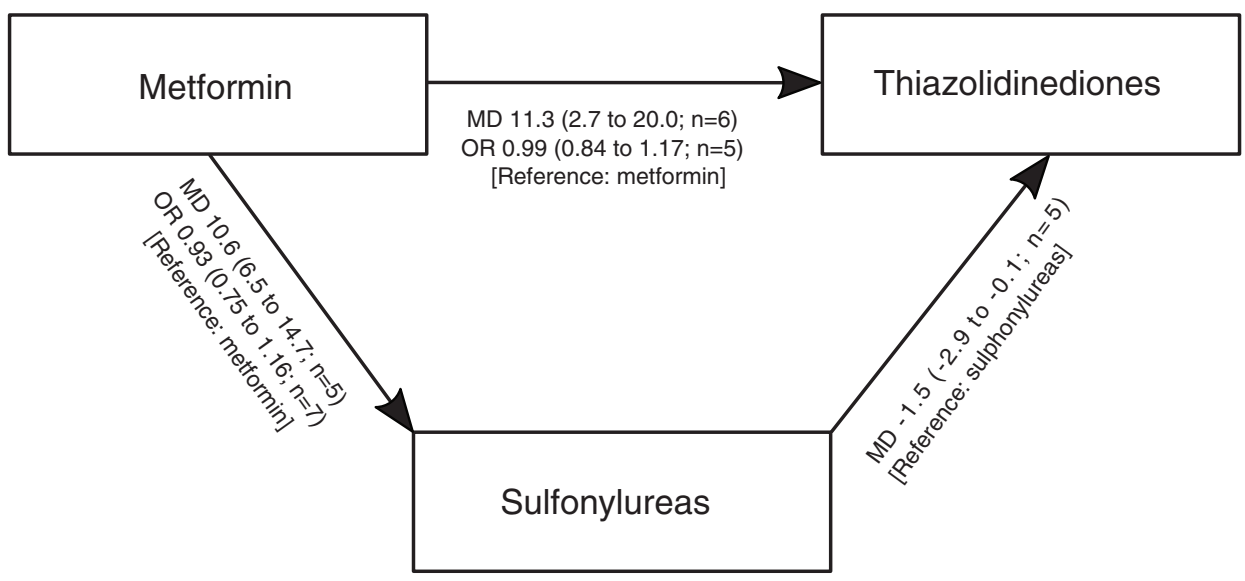

FIGURE 1 A summary of pairwise meta-analyses performed. The arrows point towards the medication which has the highest adherence or longest persistence (although not all results reach significance). Data in black are comparisons of adherence. MD, mean difference in the level of adherence when reported as proportion of days covered or medication possession ratio; OR, odds ratio for non-adherence. Data in red are comparisons of medication persistence. MD, mean difference in persistence duration (in days), OR, odds ratio for nonpersistence. The medication used as the reference group is shown in square brackets for each pairwise comparison. GLP-1, glucagon-like peptide-1

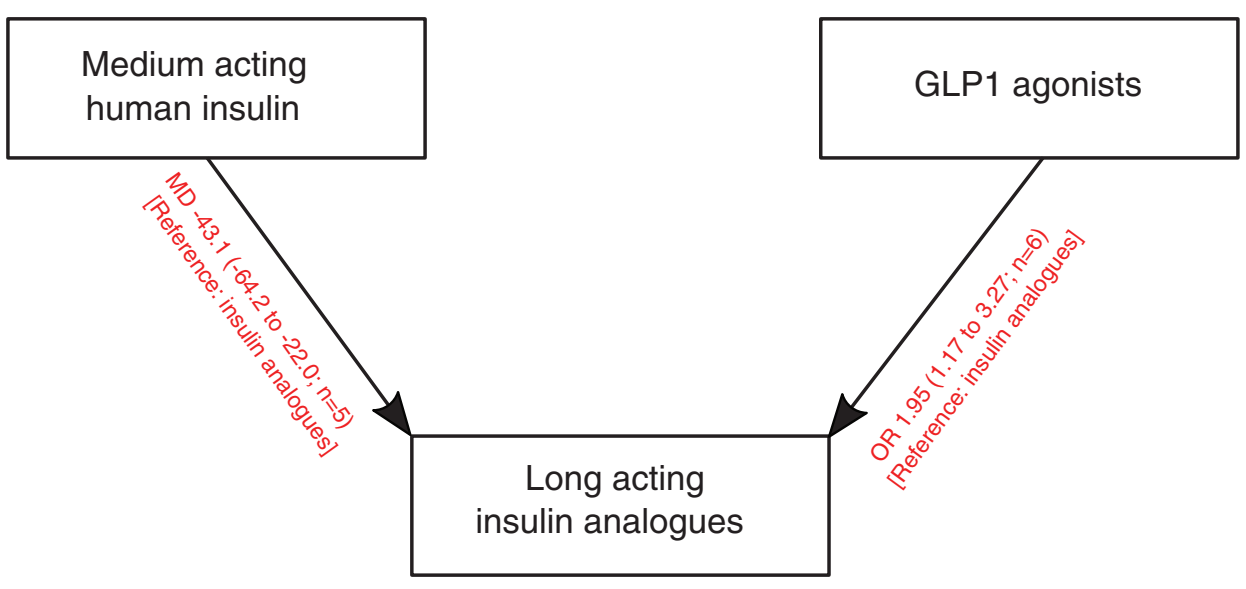

\section{4 | Quality of included studies}

The median (interquartile range) quality score of observational studies on the Newcastle-Ottawa scale was 7 (7-9), with a large proportion of studies not adjusting for potential confounders (Table S7 in File 1). The risk of bias in RCTs was moderate, with the main limitation being the lack of treatment concealment (open-label design) in almost all studies that included an injectable therapy (Table S8 in File 1).

\subsection{Definitions of adherence and persistence}

Definitions of adherence varied considerably across studies, with medication adherence reported as: mean number of missed doses; proportion of people taking $\geq 90 \%$ of doses; mean MPR or PDC; proportion of people with an MPR or PDC $\geq 80 \%$; or OR for adherence. MPR was frequently adjusted to account for differing refill sizes between products, using a predefined centile of refill time between the first and second prescription. Only 1 identified RCT reported medication adherence.

Definitions of medication persistence were even more varied. Persistence was reported as mean time to a non-persistence event, the proportion of people remaining persistent at a given time after initiation (commonly 9 months, 1 year, or 2 years), or the OR or hazard ratio of non-persistence. A non-persistence event was defined as a fixed gap between medication refills (commonly 60 or 90 days), or as an adjusted gap based on a predefined centile of refill time between the first and second prescription. Time to treatment modification (discontinuation, switching or augmentation) was also reported in some studies and, again, definitions were very variable.

\section{4 | DISCUSSION}

Substantial class differences in adherence and persistence were identified; adherence to metformin was lower than to sulphonylureas and TZDs, adherence to DPP-4 inhibitors was better than to sulphonylureas and TZDs, and persistence with long-acting insulin analogues was better than with GLP-1 receptor analogues and medium-acting human insulins. Methods for defining adherence and persistence were highly variable and all meta-analyses had a high degree of heterogeneity.

Reasons for the interclass differences observed were not reported by most studies except that several studies described the higher rates of adverse events with GLP-1 receptor analogues. It is likely that relative frequency of adverse events is also partly responsible for the lower adherence to metformin and the higher adherence to DPP-4 inhibitors. Metformin commonly results in gastrointestinal disturbance, whereas DPP-4 inhibitors are generally well tolerated. ${ }^{11}$ Multiple daily dosing may also be partly responsible for adherence differences, particularly contributing to the reduced adherence to meglitinides and $\alpha$-glucosidase inhibitors.

The primary limitation of existing adherence and persistence research is the ongoing lack of a universal definition of adherence and 
persistence. Most studies use prescription records to infer medication use and yet there is a huge variety in the methods used. This limits the comparability of studies and increases the risk of selective reporting.

The use of MPR and PDC by most studies is also a limitation, with few studies providing a direct measure of medication use; however, where both indirect and direct measures were used to make the same comparison, results were generally consistent.

Several important comparisons were not found: very few studies compared adherence across different injectable therapies. This may be because adherence is difficult to calculate from prescription records. Most insulin and GLP-1 receptor analogue studies only considered treatment persistence. There were also limited data available on comparative adherence to SGLT2 inhibitors.

The vast majority of studies were funded by pharmaceutical companies. This may increase the risk of bias towards results favouring newer pharmacotherapies, although we were not able to assess this.

The most recent guidelines for T2D management developed by the American Diabetes Association (ADA) and European Association for the Study of Diabetes (EASD) give equipoise to a range of second-line therapies. ${ }^{11}$ Given this equipoise, medication class switching may be an effective method for improving adherence in people with poor adherence. Interventional studies should assess this possibility. We suggest that class selection should consider future adherence and persistence. Where all else is equal, medication classes with better adherence (such as DPP-4 inhibitors and analogue insulins) should be preferred.

Standardized definitions of medication adherence and persistence are required. Reporting guidelines for medication adherence or persistence studies may also improve study quality.

In conclusion, adherence varies considerably across different medication classes used for the treatment of T2D. Metformin has lower adherence than sulfonylureas and TZDs. DPP-4 inhibitor adherence is better than both sulfonylureas and TZDs. Long-acting insulin analogues have better persistence than GLP-1 receptor agonists and human insulins. Preferential selection of these medication classes with better adherence and persistence may produce better outcomes in T2D. This is likely to be of greater importance in people known to have low adherence rates.

\section{Acknowledgments}

The authors would like to acknowledge the support of project manager Dr Filipa Ferreira.

\section{Conflict of interest}

AMcG has received research funding from AstraZeneca and Eli Lilly and Company. ZT has no conflicts of interest to declare. $\mathrm{WH}$ has received research funding from Eli Lilly and Company. NM has received fees for serving as a speaker, a consultant, or an advisory board member for Allergan, Bristol-Myers-Squibb, Astra-Zeneca, GlaxoSmithKline, Eli Lilly, Lifescan, MSD, Metronic, Novartis, Novo Nordisk, Pfizer, Sankio, Sanofi, Roche, Servier, Takeda. MW has received speaker fees from AstraZeneca and research funding from Eli Lilly and Company. SdeL has received research funding from AstraZeneca, Eli Lilly and Company, GlaxoSmithKline, and Takeda.

\section{Author contributions}

AMcG designed the review with critical appraisal from ZT, WH, NM, $M W$ and SdeL. AMcG and ZT conducted the searches, and extracted data from the included studies with adjudication from MW. AMcG and ZT performed the assessment of study quality and AMcG performed the statistical analysis with critical appraisal from NM, MW, and SdeL. AMcG drafted the manuscript with review and additions from ZT, WH, NM, MW, and SdeL.

\section{ORCID}

Andrew McGovern (D) http://orcid.org/0000-0002-6833-9399

\section{REFERENCES}

1. Egede LE, Gebregziabher M, Dismuke CE, et al. Medication nonadherence in diabetes: longitudinal effects on costs and potential cost savings from improvement. Diabetes Care. 2012;35(12):2533-2539.

2. McGovern A, Tippu Z, Hinton W, Munro N, Whyte M, de Lusignan S. Systematic review of adherence rates by medication class in type 2 diabetes: a study protocol. BMJ Open. 2016;6(2):e010469.

3. Currie CJ, Peyrot M, Morgan CLL, et al. The impact of treatment noncompliance on mortality in people with type 2 diabetes. Diabetes Care. 2012;35(6):1279-1284.

4. Asche C, LaFleur J, Conner C. A review of diabetes treatment adherence and the association with clinical and economic outcomes. Clin Ther. 2011;33(1):74-109.

5. Cramer JA. A systematic review of adherence with medications for diabetes. Diabetes Care. 2004;27(5):1218-1224.

6. Iglay K, Cartier SE, Rosen VM, et al. Meta-analysis of studies examining medication adherence, persistence, and discontinuation of oral antihyperglycemic agents in type 2 diabetes. Curr Med Res Opin. 2015;31(7):1283-1296.

7. Sabaté E. Adherence to Long-Term Therapies - Evidence for Action. Geneva, Switzerland: World Health Organisation; 2003. http://www. who.int/chp/knowledge/publications/adherence_report/en/. Accessed November 30, 2017.

8. Yeaw J, Benner JS, Walt JG, Sian S, Smith DB. Comparing adherence and persistence across 6 chronic medication classes. J Manag Care Pharm. 2009;15(9):728-740.

9. Cramer JA, Roy A, Burrell A, et al. Medication compliance and persistence: terminology and definitions. Value Health. 2008;11(1):44-47.

10. Aronson JK. Compliance, concordance, adherence. Br J Clin Pharmacol. 2007;63(4):383-384.

11. Inzucchi SE, Bergenstal RM, Buse JB, et al. Management of Hyperglycemia in type 2 diabetes, 2015: a patient-centered approach: update to a position statement of the American Diabetes Association and the European Association for the Study of Diabetes. Diabetes Care. 2015; 38(1):140-149.

\section{SUPPORTING INFORMATION}

Additional Supporting Information may be found online in the supporting information tab for this article.

How to cite this article: McGovern A, Tippu Z, Hinton W, Munro N, Whyte M, de Lusignan S. Comparison of medication adherence and persistence in type 2 diabetes: A systematic review and meta-analysis. Diabetes Obes Metab. 2017;1-4. https://doi.org/10.1111/dom.13160 